\title{
Cuba's Pedro Kourí Tropical Medicine Institute: Battling COVI-19 One Study, One Test, One Patient at a Time
}

\author{
Gisele Coutin MD MS, Jorge Bacallao-Gallestey PhD DSc, Lila Castellanos-Serra PhD DSc
}

This MEDICC Review roundtable brings you specialists from Havana's Pedro Kourí Tropical Medicine Institute (IPK), who are working directly with testing, research and patient care during the COVID-19 pandemic. Founded in 1937 by its namesake, the Institute has gained considerable worldwide prestige. Today, it is a PAHO-WHO Collaborating Center for the Study of Dengue and Its Vector, and for the Elimination of Tuberculosis. Its main role within Cuba's health system is as the national reference center for prevention, control, management and elimination of infectious diseases, including epidemics. Its 479 workers staff

\section{Introducing our readers to roundtable participants (in order of appearance):}

Manuel Romero-Placeres MD MPH PhD is a physician with dua specialties in family medicine and epidemiology, as well as a degree in public administration and a doctorate in medical sciences. $\mathrm{He}$ also holds a master's degree in public health, concentrating on environmental health. He is full professor and senior researcher at the Medical University of Havana, and is a member of the Cuban Academy of Sciences. Dr Romero is director of the Pedro Kourí Tropical Medicine Institute (IPK).

Daniel González-Rubio MD MS PhD is an internist with a master's degree in infectology and tropical diseases, and a doctorate in medical sciences. He is full professor and associate researcher at the Medical University of Havana and chief of the Tropical Medicine Unit at IPK.

Sonia Resik-Aguirre MD MS PhD is a physician specializing in microbiology with a master's degree in virology and a doctorate in medical sciences. She is full professor and senior researcher at the Medical University of Havana. Dr Resik heads the Virology Department and directs the National Polio Reference Laboratory at IPK. She has participated in numerous international collaborations, including 12 with WHO on inactivated polio vaccine. She is a member of WHO's SAGE Polio Working Group.

MEDICC Review: How has the Institute organized its work to address the challenges presented by COVID-19?

Manuel Romero: In research, we're beginning studies related to the clinical evolution, epidemiology and virology of COVID-19 in Cuba. The main research is devoted to characterizing the clinical manifestations of the disease, treatment protocols and clinical diagnosis. The virology studies are related to diagnosis, disease pathogenesis, as well as kinetics of the virus and its excretion in different samples; plus of course immunity and genetics research.

In epidemiology, we are analyzing the disease's characteristics in Cuba, its symptomatic and asymptomatic expressions, progno-
32 departments, including laboratories, research and teaching facilities, a hospital and isolation center. The IPK's hospital treats later-stage AIDS patients, while the Institute is the national reference center for attention to all HIV-positive patients and maintains the national HIVIAIDS registry, as well as registries for other infectious diseases. The institution was responsible for training the Cuban doctors who served in West Africa during the 2014-2016 Ebola outbreaks and for those going abroad to assist in the COVID-19 response today, and its professionals offer an internationally-recognized biennial course on dengue.

sis, and the influence of climatic variables. Simultaneously we are studying costs. Multidisciplinary teams of epidemiologists, sociologists, mathematicians, geographers, biostatisticians, economists and information technology (IT) specialists have been set up to carry out epidemiological surveillance and research in this arena.

To ensure 24-hour surveillance at the Institute, we have established a temporary decision-making task force, headed by an expert epidemiologist, including a resident in hygiene and epidemiology stationed at hospital admissions, plus a computer specialist for data processing. This group supports the Ministry of Public Health's own 24/7 epidemiological surveillance and participates in its working groups of epidemiologists and geographers. Thus, we send daily reports to the Ministry of Public Health (MINSAP) on admissions, discharges, suspected COVID-19 cases seen, and the epidemiological histories of the hospitalized cases. This is fundamental for contact tracing and timely control of potential transmission clusters at the primary health care level. We also publish a weekly epidemiologic bulletin on the disease to update medical personnel nationally.

In the academic area, IPK has developed a training program for our own personnel as well as those in the national health system and other government agencies involved in addressing the disease. We taught a national course on prevention and control of the new coronavirus for a group of health professionals who then served as facilitators to share the knowledge in each province; and we developed an online course as well. We are involved in three specific aspects of teaching now: care for suspected or positive cases, confirmatory diagnosis, and preparation of all personnel going abroad in the Cuban medical teams serving in other countries to help confront the pandemic.

IPK's hospital is in quarantine, devoted to care for suspected or positive COVID-19 patients. There, we have a triage admissions unit, where adults and children are seen separately. There are 7 hospitalization wards with 70 two-bed rooms, distributed as follows: one for pediatric and obstetric patients (suspected positive); two for confirmed cases (red zone); and four for other suspectedpositive patients. We have an ICU for serious-to-critical patients 
or those who require closer monitoring. Outside our facilities, we also supervise the Antonio José de Sucre Isolation Center in Jagüey Grande, Matanzas Province, which is also used as a quarantine center for COVID-19.

We have the human and material resources we need to care for these patients, including molecular diagnosis (real-time polymerase chain reaction; RT-PCR), and personal protective equipment (PPE) for our workers. IPK and Cuba as a whole have received donations from $\mathrm{PAHO}$; the governments of China, Russia and Venezuela; national and international scientific institutions; joint enterprises here in Cuba; Cuban farmers and other individuals; and Cuban non-governmental organizations, among others.

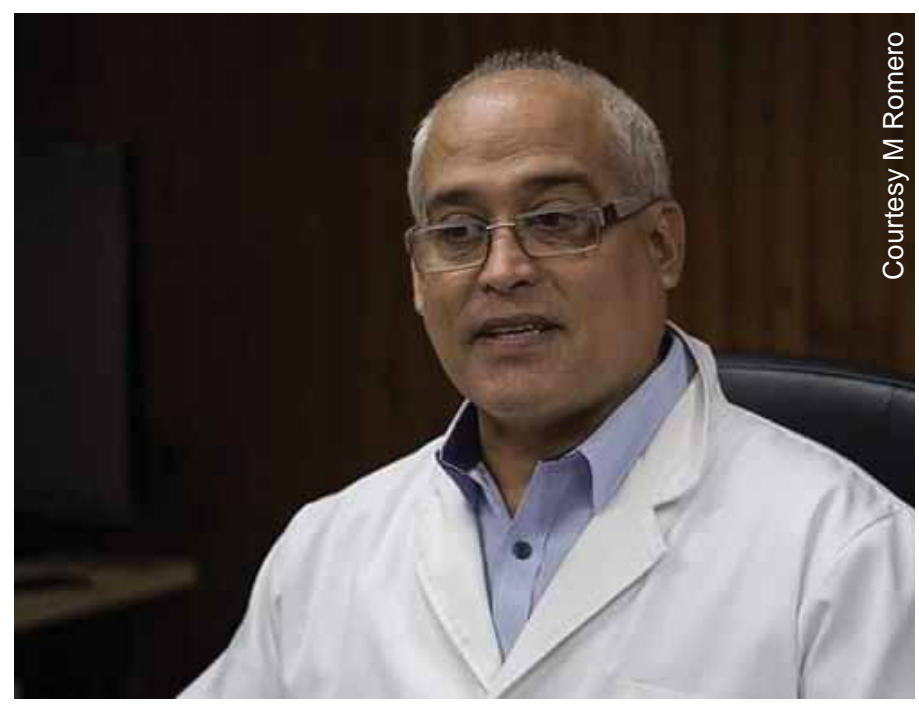

Manuel Romero: We send daily reports to the Ministry of Public Health on admissions, discharges, suspected COVID-19 cases seen, and the epidemiological histories of the hospitalized cases.

Daniel González: On the clinical front, we have a group of internists, who have either master's degrees or doctorates in infectious diseases, as well as critical care specialists, all selected from among IPK's main clinical experts. This group guides all COVID-19 case management, whether confirmed or suspected, based on the attending physicians' reports. The attendings are primarily internists (with master's degrees in infectious diseases and/or tropical diseases), plus family doctors and other physicians in these master's programs. For serious and critical cases, we also consult with a national group of critical care specialists who play an advisory role throughout the country.

Our IPK clinical team interconsults with the central group headquartered here comprising epidemiologists, virologists, biosafety experts, managers and MINSAP representatives. They discuss the clinical-epidemiologic aspects case-by-case, as well as the situation of serious and critical patients, results of complementary analyses, candidates for discharge, and follow-up strategies for those who have been released.

Here in Cuba under MINSAP guidance, each death is exhaustively discussed at the national level, reviewing the therapeutic decisions made at each stage of treatment and the patient's response to them.
MEDICC Review: How has COVID-19 most frequently presented in the patients in your care?

Daniel González: COVID-19 is an emerging disease, with only a few months on the global radar. Despite this, it has been well characterized clinically. One quarter of confirmed patients arriving at IPK were asymptomatic, resulting from tracing contacts of suspected or confirmed cases. With few exceptions, they have come to us in the disease's initial stages, and any complications have started between the fifth and the eleventh day after symptoms first appeared. Among those who had symptoms, most are mild and their cases uncomplicated: most frequent symptoms are dry cough $(85 \%)$ and fever (65\%). Most symptoms last for three to five days. Nevertheless, $15 \%$ of patients present with serious pneumonia.

I want to note the special attention being paid to risk groupssuch as older adults, pregnant women, patients who have cancer or are immunocompromised-which begins in primary health care. Two related elements of the Cuban approach are important here: active case detection and quarantine in isolation centers of all contacts of positive patients. I believe few countries could do something similar.

MEDICC Review: What are the essential elements for your diagnosis of COVID-19? And what role has IPK played in establishing diagnostic criteria nationally?

Manuel Romero: When SARS-CoV-2 appeared, one of our experts from IPK's National Virology Reference Laboratory received PAHO-sponsored training for diagnosis by RT-PCR, and we immediately introduced this at IPK.

Actually, once Zika hit our radar a few years ago, and with support from a research project in collaboration with $\mathrm{WHO}$ and MediCuba-Europa, MINSAP established labs for molecular diagnosis in the Provincial Hygiene and Epidemiology Centers in Havana and Santiago de Cuba, later adding Villa Clara Province, equipped with advanced technology. IPK trained all these labs' personnel, and later we incorporated the Molecular Biology Laboratory of the Civil Defense's Scientific Research Center, as well as those of the Genetic Engineering and Biotechnology Center and the Hermanos Ameijeiras Clinical-Surgical Teaching Hospital in Havana.

Moreover, the National Virology Reference Laboratory (NVRL) at IPK is responsible for quality control of diagnoses made in all these centers, so there is constant exchange with these laboratories and our own.

Sonia Resik: With this kind of disease that can hit anyone, virology diagnosis is essential, so very early we started special training of our laboratory staff under PAHO auspices. We also participated in conceiving the national plan to confront the disease and in designing guides for taking samples for virology diagnosis, in biosafety training, and in the molecular diagnosis techniques we would be using across the country. Two of our virologists are also abroad, assisting other countries in the region.

Manuel Romero: The NVRL is working around the clock to provide results within 24 hours of receiving samples. Its staff is working in shifts: 24 hours on, 72 hours off. In addition to quality control, the NVRL is responsible for molecular diagnosis of our 
own patients and all those of suspected cases in the western provinces of Pinar del Río, Matanzas, Mayabeque, Artemisa and the Isle of Youth Special Municipality.

MEDICC Review: Globally we have seen very rapid disease progression in some patients, particularly those advancing to serious or critical stages. What criteria do you use for evaluating patients, and have you developed tools to anticipate which ones may have a worse prognosis?

Daniel González: Yes, the disease's course varies widely and many times unpredictably. We know of cases where a person has progressed from a practically asymptomatic status to respiratory distress within hours. For any physician, this is disconcerting and very troubling; we've commented that we have never seen such a thing before.

Luckily, such evolution is infrequent. In our own experience and in the international literature, we have found certain clinical, radiological and laboratory elements precede severity and can alert us to potential decline. So this is one of the pillars of our protocols: early identification of these factors, to get ahead of severe evolution.

On the clinical side, a key sign is dyspnea: that is, before full manifestation, patients describe feeling tired or "different" while carrying out normal daily functions like bathing or brushing their teeth. Physicians can detect a slight increase in respiration rate, while digital oximetry will show slight oxygen desaturation. Auscultation may reveal presence of some moist, crackling rales, still without fully established pneumonia. A chest x-ray can corroborate such physical exam findings, revealing initial stages of pneumonia. Also related to severity are increased blood levels of lactate dehydrogenase (LDH), transferrin, reactive $\mathrm{C}$ protein and globular sedimentation velocity. Other factors are leukopenia accompanied by lymphopenia and thrombocytopenia.

Such findings enable timely intervention to minimize risk of patients progressing to severity, and we have applied this approach

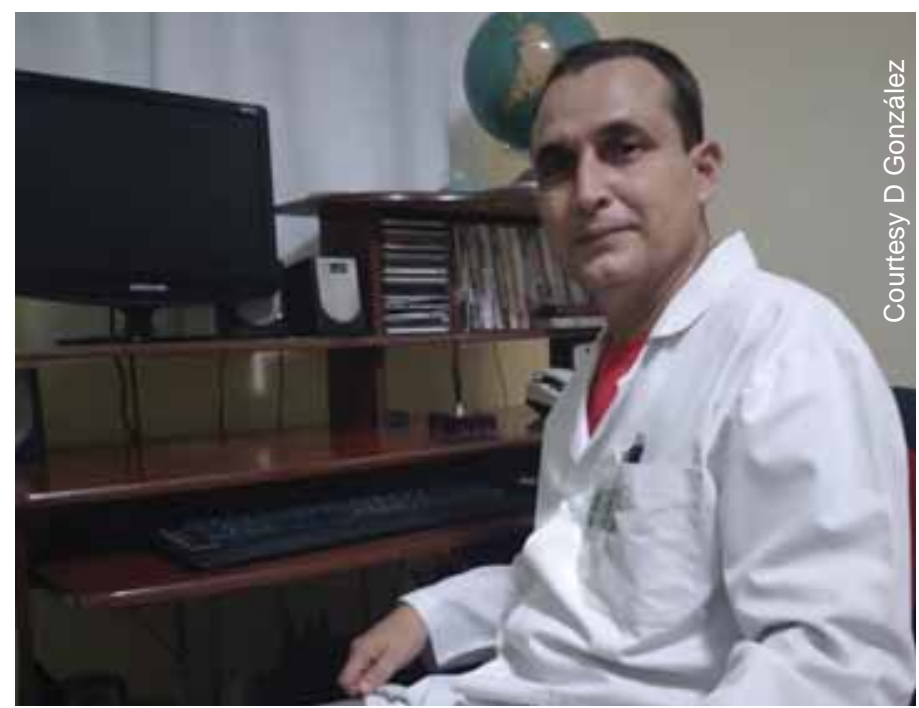

Daniel González: We know of cases where a person has progressed from a practically asymptomatic status to respiratory distress within hours. For any physician, this is disconcerting and very troubling. with good results. Another important aspect is applying treatment protocols as early as possible. In fact, we recommend initiating treatment before COVID-19 confirmation in patients highly suspected of infection or with risk factors.

\section{MEDICC Review: What treatment protocols are you following?}

Daniel González: We follow protocols guided by international experience, adjusted to our own. Protocols vary, depending on whether the patient is an asymptomatic contact, a suspected case or a confirmed case. For confirmed cases, we combine antiviral medications with immunomodulators and broad-spectrum antibiotics (if they develop bacterial infections), carefully monitoring for other complications that might arise. There are protocols for managing respiratory distress and other events such as sepsis, shock and organ damage. The medications we use in Cuba coincide with those included in other countries' protocols, and we apply them nationally in all institutions.

Another characteristic of Cuban protocols is inclusion of novel medications developed by our biotech industry, such as Biomodulina $\mathrm{T}$ and the pulmonary surfactant Surfacén, manufactured by the National Center for Bioproducts, and the Itolizumab monoclonal antibody from the Molecular Immunology Center. We are also using nationally-produced interferon alfa-2b, of course.

MEDICC Review: In some countries, plasma from recovered COVID-19 patients is being used for seriously ill patients who have not responded to other treatments. What is your experience, your perspective on this?

Daniel González: Cuban experts are working under the principle that no hypothesis should be rejected out of hand concerning the disease's pathogenesis or therapeutic options. We analyze all the information we receive. Plasma transfusion from convalescing patients is an alternative with some unanswered questions. It's true that it has been used quite successfully for other infectious diseases. To use it, two essential components must be considered: the donor and the recipient.

For COVID-19, there are still doubts about when a donor is completely "cleared" of infection, because we know of some cases of disease recurrence. On the receiving end, these patients usually develop a serious case in the second week of evolution, that is when they have already developed their own antibodies to the virus. We don't know to what extent those patients might benefit from introducing other antibodies.

Nevertheless, I reiterate that this is an alternative for treating a disease that daily is taking thousands of human lives, and thus, all therapeutic alternatives should receive consideration.

MEDICC Review: What criteria do you use to discharge a COVID-19 patient? And what about immunity conferred, reinfection or possibilities of remaining contagious?

Daniel González: Discharge criteria are well defined and include clinical, epidemiological and microbiological elements. The main clinical criterion is that the patient must be free of major symptoms that could be life-threatening; and epidemiologically, must be symptom-free for at least 14 days. Additionally, absence of SARS-CoV-2 must be confirmed by RT-PCR. All three criteria 
must be met for a patient to be discharged. Then they must spend another 14 days in home quarantine, under supervision by physicians in primary health care.

Some aspects of COVID-19 remain unclear: possible re-infection or relapse, immunity, the potential time the disease is contagious or its capacity to infect other hosts and additional transmission routes besides respiratory. Nevertheless, we think the criteria we're applying for medical discharge and posthospitalization surveillance minimize risks for the patient, their family and their community.

MEDICC Review: How are you dealing with the understandable fear and anguish in these patients, especially those who are seriously ill?

Daniel González: This is a very tough challenge. The pandemic is rightfully receiving broad media coverage, but this can also create more anguish in patients suffering from the disease. A good, harmonic doctor-patient relationship is essential for psychological management. But this relationship is seriously compromised by various factors inherent in COVID-19's case. Patients think they may die, and that in itself generates anguish and can even lead to panic. What's more, they are hospitalized in total isolation, with no visitors, not even able to leave their rooms.

At the same time, doctors have to see their patients while fulfilling biosafety requirements, which creates a physical barrier between them and their patients. In this adverse scenario, doctors must make their maximum effort not only to provide the best treatment possible, but also to provide the equally important psychological support. In this, nursing staff is fundamental, especially because they have frequent contact with patients. Their selflessness and dedication, their humanistic values, are constantly being tested, in a role where their technicalprofessional expertise must go hand-in-hand with compassion, while taking strict care of themselves as well.

When a patient dies, informing their relatives is a terribly difficult moment, both for the families and for the doctors who have to give them the news. We always hold out hope, even for critical patients, but it is painful to the extreme when the virus wins, even after applying all the therapies at our command and making all possible efforts to save them.

MEDICC Review: We know that IPK has well-established protocols for protecting its personnel when addressing infectious diseases. Have you taken additional measures with COVID-19?

Manuel Romero: As mentioned, IPK's hospital is in quarantine. We have established three staff groups, each comprising doctors, nurses, lab technicians, radiologists and support staff. They rotate 14 days in the hospital, 14 days in one of the isolation centers, and 14 days at home. During the 14 days here, we abide by standard labor codes in place in terms of hours and breaks, and they have adequate conditions to rest. To protect our personnel, we follow strict biosafety regulations, with PPE determined by the degree of risk in each zone where people work, and we constantly monitor fulfillment of biosafety measures.
A special protocol was established at IPK for management of suspected and confirmed COVID-19 cases, and we trained nurses, doctors and service staff, including housekeeping, kitchen staff and those who distribute food trays. Each work area is monitored daily by the biosafety department. For example, we have established: biosafety measures in areas of admissions and patient care, with access determined by the type of area; instructions for personal hygiene and cleaning of each area; measures for manipulating samples, food, waste, hospital linens and protection equipment; norms for disinfecting and sterilizing intensive care units; indications for quarantine; and organization of regular health and safety inspections.

MEDICC Review: As a virologist with more than 30 years' experience, what new challenges has this pandemic posed?

Sonia Resik: Many! First, how to lead a group of scientists and young researchers, motivating them to give their all under conditions completely different than those they are used to. Confronting the newness of this disease, an infectious disease not seen before, demands the best in terms of human and professional values.

This isn't the first epidemic we have addressed or have prepared for. There have been many: dengue, Zika, epidemic neuropathy, H1N1 influenza, SARS, hemorrhagic conjunctivitis, HIVIAIDS, Ebola. In some cases, these diseases haven't reached Cuban shores, but in others they have had a serious impact on our people's health.

But this pandemic is different from all the others because COVID-19 is highly contagious, the SARS-CoV-2 virus highly transmissible. It spreads very rapidly, and in less than two months was in almost every country in the world. Emerging and re-emerging diseases have a very important social component as well, given the economic and social factors that facilitate their advance. Globalization is also to blame for this phenomenon: we travel from one part of the world to another in mere hours, and viruses and other microorganisms accompany us. And since this is an emerging vi-

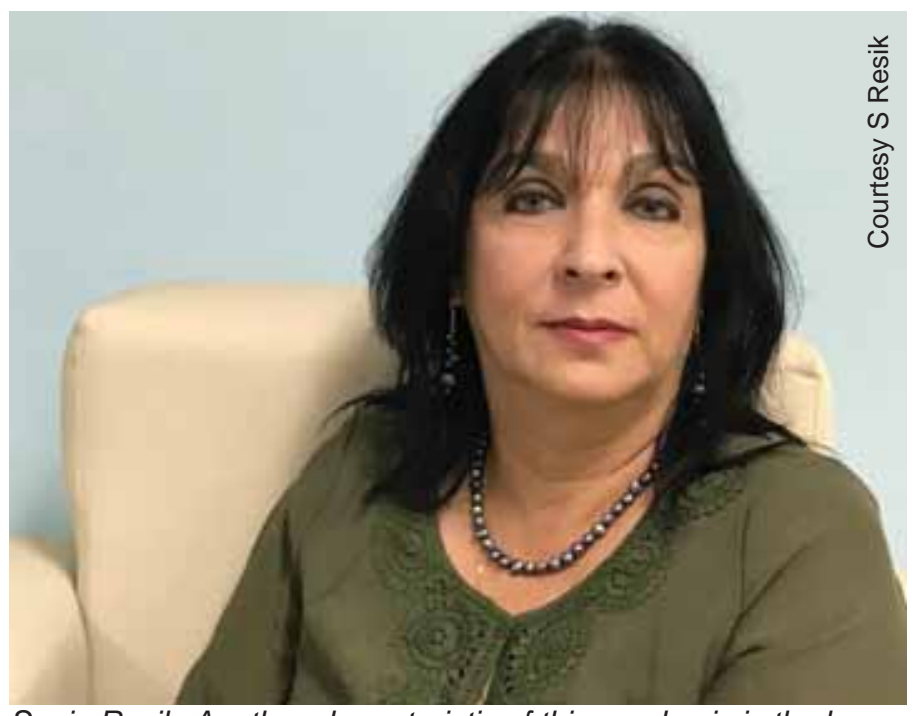

Sonia Resik: Another characteristic of this pandemic is the large number of asymptomatic patients. They are infected, transmit the virus, but don't have symptoms to permit clinical diagnosis. 
rus, it finds a "virgin" population to colonize. There is no previous immunity, and so we are all susceptible to contracting the disease. The high levels of environmental contamination, megacities with overcrowded neighborhoods, poverty and inequality are all factors that make for greater risk of pandemics.

Another characteristic of this pandemic is the large number of asymptomatic patients. They are infected, transmit the virus, but don't have symptoms to permit clinical diagnosis. As we say, "this virus has no face." This is why strict measures of physical distancing and personal responsibility are so important, as well as increasing people's risk perception. And we have to focus particularly on young adults, who most often are responsible both for children and the elderly.

Another challenge right now in this global context is availability of resources, in our case aggravated by the constant US blockade. Although we don't have an abundance of material resources, we do have the most important thing for confronting the pandemic: our human resources, their sense of service, solidarity and sacrifice for the good of others. As scientists, we have another challenge: to be altruistic by sharing information. All scientific information that we can collect concerning this disease must be for the world, for the collective good of everyone, because COVID-19 is a global problem that affects all of us.

MEDICC Review: What lessons-from IPK's experience and your own personal experiences-would you like to share with our readers?

Manuel Romero: At IPK, specialists are keeping records of our daily activities, anything that might be of interest, to document the experience we're accumulating. Since March 11, 2020, when we diagnosed the first cases, we've been keeping complete statistical data as well. This recordkeeping is supervised nationally by MINSAP, which has also created a system for documenting and preserving the country's experience confronting the COVID-19 pandemic.

Sonia Resik: We have to overcome the fear of the unknown, something new. We've all felt it. But continuous learning and our experience help us confront it. This kind of fear also helps us to adapt our behavior to the situation, for our own benefit, in this case to protect ourselves and the people around us.

And I certainly haven't escaped that fear, especially when my son and daughter-in-law, both young internists, told me they were coming to IPK to work with COVID-19 patients in the "red zone." As a mother, I was afraid, but as a physician, I had to overcome that fear, because I'm also working in virologic diagnosis of these patients. And ever since he was born, my son has seen me working, studying, studying some more, facing epidemics. So right now, I can't let that mother's fear restrict him. And all three of us are dedicated to helping our population survive this pandemic, to maintaining the free and universal health care we have had for the last 60 years.

You also have to face distancing from other family members, taking special care if they're older. That's the case of my own parents. They understand I have to be at work, that their grandson-who they adore-has to be here, too. I talk with them by phone every day. Meanwhile, my sister and brother-in-law pick up the rearguard to attend to my parents, since everyone understands the urgency of the situation.

I say medicine is a vocation, a calling, and it's for life. Because the point is to guarantee everyone from birth the right to health care, and the best care possible. That's why we're here.

Daniel González: We've all lived through many days and many experiences we could talk about. A few days ago, one shift of workers who were finishing up their 14 days on duty asked me: "Professor, why substitute us? We're not tired yet!" That kind of willingness on the part of such a young staff fills you with pride, especially because most of them were trained at IPK. Another time I went to console a critical care doctor who had just lost a patient and was crying bitterly as if she had lost a member of her own family. Standing outside to send off a recovered patient who is being released is really something, a feeling I'll never forget. Personally, one of my most gratifying times is looking over the daily emails I get from my regular patients, full of encouragement, and asking me to take care of myself. What a treasure! -1 - 